\title{
A study of the coarse water droplets formation in the nozzle
}

\author{
Lucie Měšt’anová, and Ondřej Bartoš * \\ CTU in Prague, Department of Energy Engineering, Technická 4, Prague 6, 166 07, Czechia
}

\begin{abstract}
The paper introduces the experimental results of the droplets formation for three different aqueous solutions. The new wind tunnel was built to study water droplets atomization from liquid films at high speed flow similar to that found in steam turbines. Liquid atomization is a widely studied problem for sprays and generally in the field of aerosol research. A similar phenomenon occurs in steam turbines but mainly with undesirable effects, the formed droplets from the film (coarse droplets) have a negative effect on the reliability and efficiency of the turbines due to the erosion and corrosion by the droplets impact on the leading edges of the blades. The new wind tunnel is equipped with classical pressure and temperature measurement systems for the determination of the initial condition in the settling chamber and measurement of the static pressure along the nozzle with a known profile. The photogrammetric method and light scattering are used to measure the diameter distribution of the droplets. The liquid film is made with an aqueous solution supplied on the symmetrical aerofoil NACA 0008. Different aqueous solutions were studied for different liquid surface tension effects.
\end{abstract}

\section{Introduction}

The paper contains the first result of the measurement of three different aqueous solutions with different surface tensions. The purpose of the measurement was to assess the surface tension effect on the atomization of the liquid film.

The atomization of liquid is a widely studied problem for spray nozzles and in the field of aerosol research. A similar phenomenon is known in steam turbines but mostly with undesirable effects, coarse droplets have a negative effect on the reliability and efficiency of steam turbines. The study of the water droplets formation, movement and further impact back on the moving blade in turbines at high speed has an important role in the never-ending effort to enhance the efficiency of the energy production and decrease its environmental impact. There are other applications where liquid films are present on a moving body, for example on the trailing edge of wings, propellers and blades the result of this study could be useful. Nevertheless, due to the wide range of applications the project is focused on the conditions in steam turbines. In the long term the Department of Energy at CTU is concerned with experimental research in the liquid phase structure in steam turbines [1]. Two groups of water droplets are present in steam turbines. The nucleation of the droplets and their subsequent growth due to the surface condensation are responsible for the presence of fine droplets. The expected range of fine droplets is from $50 \mathrm{~nm}$ to $800 \mathrm{~nm}$. Coarse droplets are formed by the disruption of water films on the blades and casing surfaces in the low-pressure turbine stages. The present knowledge of the coarse water flow field and the size distribution of the droplets in turbines is still insufficient. The range of the diameters of the coarse droplets is very wide, from a few micrometres to one millimetre. The coarse droplets are responsible for some unfavourable effects like additional energy losses and a reduction in the lifetime of the blades. A better understanding of the formation of coarse droplets and their properties may provide future enhancements for the steam turbine and other similar applications. Previous measurements of coarse droplets directly in the steam turbine made it necessary to build an experimental tunnel for the study of the formation of the droplets and their properties [2] in the laboratory.

\section{Testing facility}

The motivation for the new wind tunnel assembly arises from the previous measurement [2] of the coarse droplets in the steam turbine under operational conditions. The measurement was performed in three steam turbines in the Czechia. In all power stations the amount of the acquired coarse droplets was very low due to their low number density. The diameter of the captured droplet was around $60 \mu \mathrm{m}$. The diameter of the coarse droplets according to the Weber number (eq. 1) was expected to be smaller. Due to difficulties connected with making measurements in an operating steam turbine, a new wind tunnel was designed and manufactured for the analysis of the coarse droplets formation from the liquid films. The goal of the design was to encounter the conditions in the steam turbine as closely as possible together with the advantages of working in the laboratory.

Corresponding author: ondrej.bartos@,fs.cvut.cz 
The tunnel is designed as a classical CD nozzle, but the planned operational regime is mainly subsonic or transonic. An aerofoil NACA0008 is placed $50 \mathrm{~mm}$ behind the nozzle throat (Fig. 1). The aerofoil is simulating the blade in the turbine and it is possible to replace it or even remove it. On the aerofoil is a groove to supply liquid on the surface. The tunnel is equipped with four large optical windows to provide good optical access for the measurement. The liquid is pumped to the aerofoil through the dozing pump with a flow from $1 \mu \mathrm{L} / \mathrm{min}$ to $500 \mu \mathrm{L} / \mathrm{min}$. This range is sufficient for the simulation of different liquid films on the aerofoil. It is possible to operate the tunnel with steam in continuum mode or with compressed air for periodic measurements. The measurement schema is shown in Fig. 2.

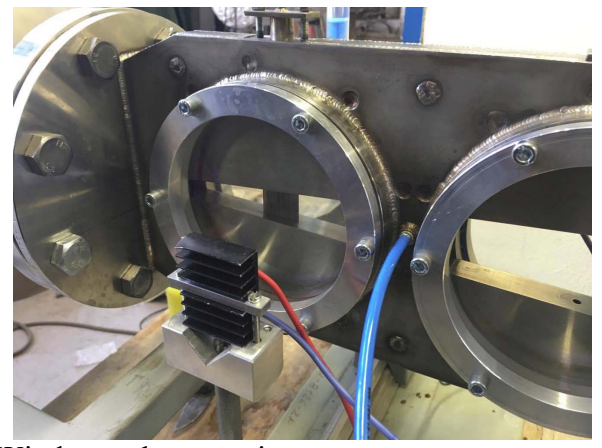

Fig. 1. Wind tunnel test section.

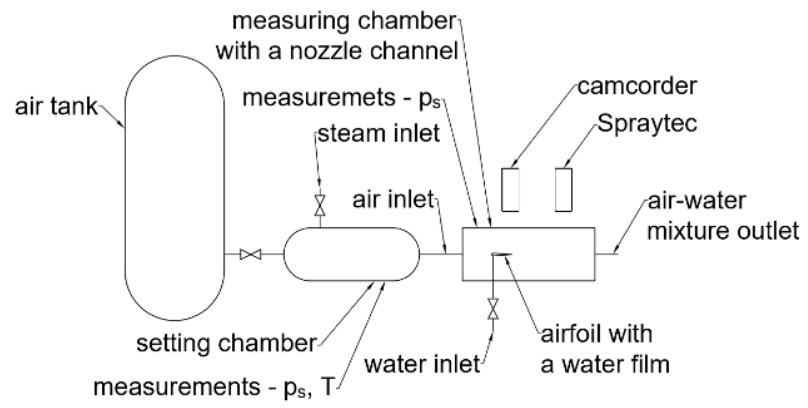

Fig. 2. Measurement schema of the nozzle.

\section{Measurement method}

The time duration of the measurement in the wind tunnel with air depends on the pressure in the compressor storage vessel. The pressure and temperature of the entire measurement sequence is acquired by the NI SCXI measurement system for the description of the flow condition for each measurement.

The measurement of the droplets size is divided using two methods. For small particles, the light scattering method is used and for bigger droplets and for droplets formation on the aerofoil trailing edge the photogrammetric method is used.

The expected diameter of the coarse droplets in steam turbines is ranges widely from 1 to $1000 \mu \mathrm{m}$ and a similar range is expected for the nozzle. This range of diameters is almost impossible to measure with one single optical technique. For that reason the new wind tunnel is equipped with the two main optical methods, photogrammetry and light scattering. These methods can cover most of the range with sufficient overlapping.

Photogrammetry is a method for determining the object properties from a picture captured by a camera. The theoretical resolution of the method is determined by the pixel pitch and the magnification of the objective lens. The size of the pixel pitch for the XIMEA camera used with a SONY CMOS sensor is in the range of $3.45 \mu \mathrm{m}$. The magnification of the objective is limited by the minimum exposure time of the camera when the global shutter is used, the measurement was made with a $2 \mathrm{x}$ magnifying telecentric objective. Higher magnification can provide better resolution for smaller droplets, but the displacement of the droplet may then be bigger than the size of the observed volume when the picture is taken. The velocity of droplets can reach more than $300 \mathrm{~m} / \mathrm{s}$ in the turbine and in the wind tunnel it is possible to reach even higher velocities. The droplet velocities and the minimum exposure time define the size of the observed volume. The entire observed volume has to be imaged on the camera sensor and this requirement limits the magnification of the objective. This means that each droplet is represented on the picture as a line. The length of the path and the exposure time allows for computation of the velocity of the droplet and determines the expected direction of the droplet's movement. But it is necessary to realise that the view of the motion is two dimensional. This means that one can only see the components of the velocity perpendicular to the axis of the camera. The depth of the observed volume is determined by the depth of field of the objective. A telecentric objective provides the advantage that the image size is independent of the distance from the objective. Calibration is required for the correct pixel/dimension ratio, for the camera used and the objective the ratio was determined with help of the calibration target (Fig. 3) of $1,7 \mu \mathrm{m} / \mathrm{px}$. For the purpose of this measurement a new light source with variable length of the flash at the order of $5-100 \mu$ s is was developed.

The measurement based on light scattering is performed by the commercial measurement device Spraytec (Malvern Ins). The application of this method is mainly for the determination of the smallest particles formed on the aerofoil trailing edge which are under the limit of the photogrammetry method and as a redundant measurement system as well.

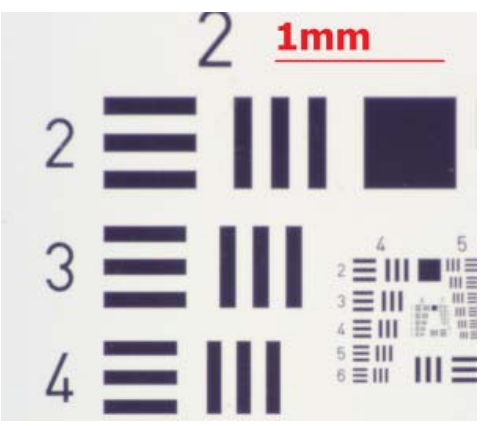

Fig. 3. Calibration target 


\section{Data processing}

The measured data provides sufficient information about the flow field in the nozzle. The images of the droplets formation show the droplet formation process and from the light scattering measurement the size distribution function of the droplets is available. One aim of the paper was to compare the formation process for liquid solutions with different surface tensions. Three selected solutions were measured separately. As the reference solution "demi" water was chosen $\sigma=0.072 \mathrm{~N} / \mathrm{m}$. The second was saturated solution of sodium chloride $\sigma=$ $0.088 \mathrm{~N} / \mathrm{m}$ and the last was water with detergent. The exact data for the particular detergent aqueous solution was expected according to the literature as $\sigma=0.045 \mathrm{~N} / \mathrm{m}$ [5]. The measurement of the liquid film breakup is still at the beginning and the expected results are more illustrative for the assessment of the influence of the surface tension during the high speed breakup.

The typically used non dimensional parameter for the droplets formation is the Weber number (1) defined as the ratio between dynamic forces and surface tension.

$$
W e=\frac{\rho_{g} w_{r}^{2} d}{\sigma}
$$

where $\rho_{\mathrm{g}}$ is the vapour density, $\sigma$ is the surface tension of water, wr is the relative velocity between the vapour and droplets, and $\mathrm{d}$ is the diameter of the droplets.

\section{Acquired images}

The results presented in this chapter are presented as an example of information for each aqueous solution. The droplets formation on the trailing edge is a chaotic process and the desired general solution requires a large number of measurements and statistical data processing for different conditions.

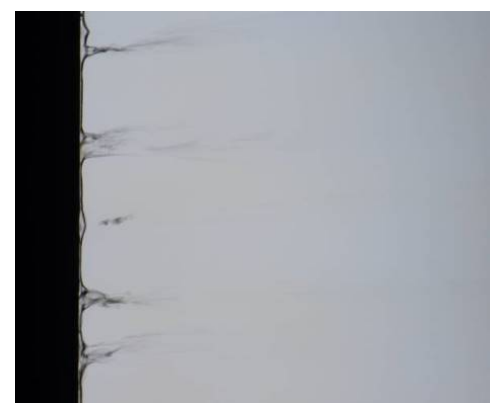

Fig. 4. The droplets formation on the trailing edge of the profile in the wind tunnel.

These measurements are not available at the moment and the current results should be considered as a promising beginning. The images (Figs. 4, 5, 6) were captured for similar conditions. The stagnation pressure in the air storage vessel was 3 bar, the flow of the dozing pump was set to $5 \mathrm{ml} / \mathrm{min}$. The observed area is $3.5 \times 4 \mathrm{~mm}$. All images show a similar process of the formation of a liquid curtain on the trailing edge. Then from the curtain liquid peeks are formed and from them the atomization to fine particles was observed

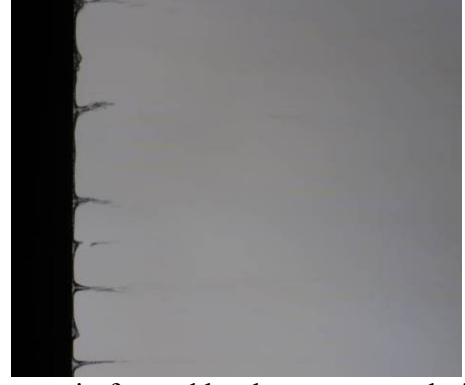

Fig. 5. Water curtain formed by the aqueous solution of the detergent.

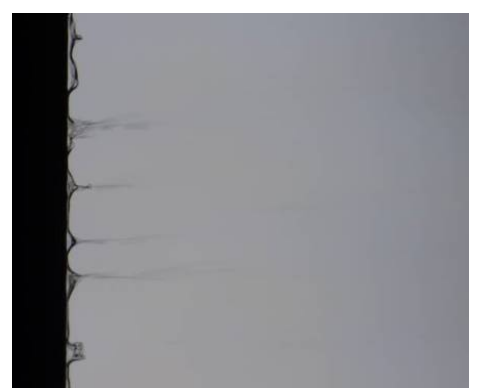

Fig. 6. Saturated solution of the sodium chloride

\section{Results}

Data processing of the collected images from the photogrammetric measurements was performed in a Matlab environment. The following figure (Fig.7) presents the analyses of the liquid amount on the aerofoil trailing edge with a dependence on time. As was mentioned in the previous part of this paper, the difference between all three substances seems to be relatively small and this requires a large number of pictures to yield reliable results. The area of the water on the trailing edge is similar for all three solutions and for the image is approx. $0.1 \mathrm{~mm}^{2}$. Image processing together with automatic edge detection was used for analysis.

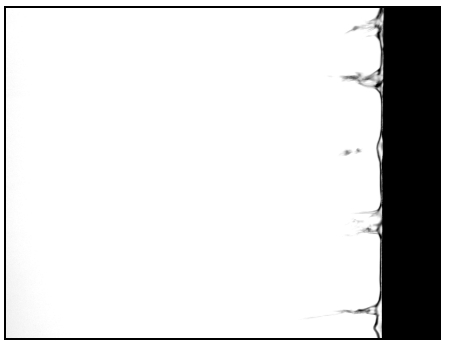

Fig. 7. An example of the liquid contours for estimation of the liquid amount on the picture.

The measurement of the size distribution function of the droplets behind the aerofoil using the Spraytec is shown in the following images Figs. 8 and 9. On the first image the volumetric distribution for all three solutions is presented and the second chart shows the number density distribution for the same measurements. Volumetric distribution with an expected constant density is the mass distribution. In Fig. 8 the bi-modal distribution is clearly visible for all the measurements. The first modus is approx. $20 \mu \mathrm{m}$ and the second $200 \mu \mathrm{m}$. 


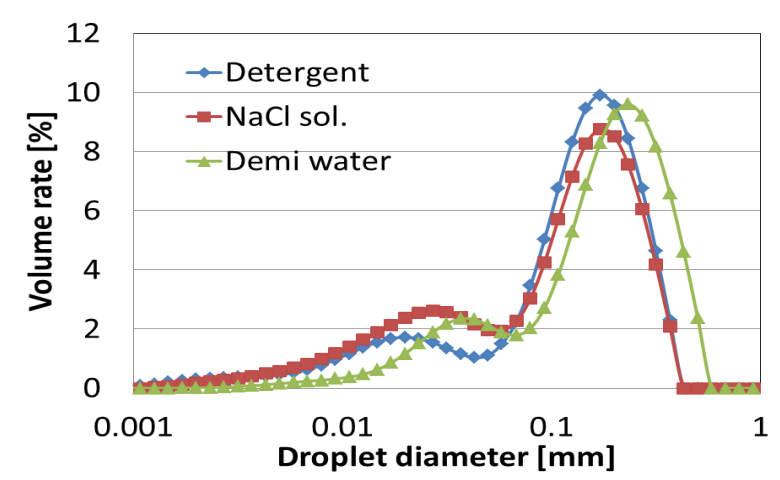

Fig. 8. A volumetric distribution of the droplets.

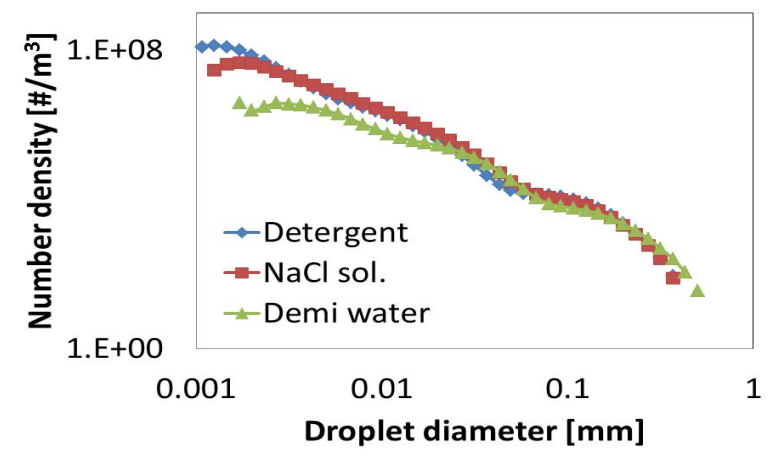

Fig. 9. A number density distribution of the droplets.

Fig. 9. shows the number density distribution. For any analysis it is necessary to realise that the number density is very different compared to the mass distribution. The observation of the coarse droplets formation is in good agreement with the measurement.

Due to the existence of bimodal distribution, the data of the size distribution function was divided into two groups and for each of these the Sauter mean diameter $\left(D_{32}\right)$ was computed. The edge diameter between the small and big droplets was estimated at $60 \mu \mathrm{m}$. In Fig. 10 the time dependence of the

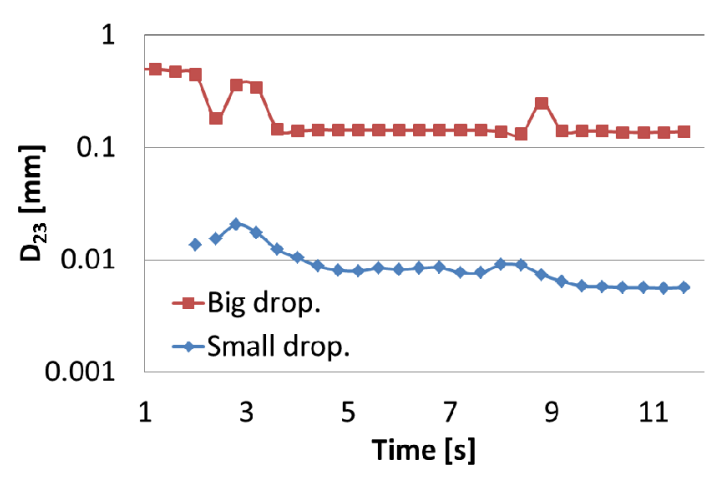

Fig. 10. Time evolution of the Sauter mean diameter for measurement with detergent.

Sauter diameter of both groups acquired from the measurement with Spraytec can be observed. In the case of this measurement, the Weber number for the first group is around $\mathrm{We}=10-25$ and for the second modus around $\mathrm{We}=100-250$. The Weber number is determined with respect to the velocity profile in the nozzle. Fig. 11 shows the profile of the pressures and velocity during the typical measurement. The velocity is almost constant during the measurement due to aerodynamics blockage in the throat of the nozzle.

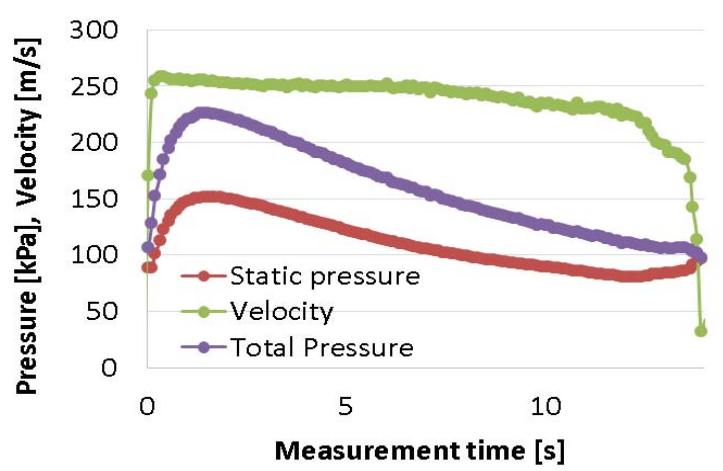

Fig. 11. The typical profile of the stagnation and static pressure and velocity profile around the aerofoil.

\section{Conclusions}

In previous works $[3,4]$ a critical Weber number was found which indicates the biggest droplet formed by the gas liquid interaction for a higher Mach number of approximately 40 . The required data about the diameter of the droplets for all three aqueous solutions disagrees with the commonly used estimation with the Weber number. In general, the disagreement increases with the velocity. In this work new knowledge about the bimodal distribution of the formed droplets was used for additional data processing. The effect of the surface tension seems to be weak in comparison with the dynamic forces. Further study of the droplet formation on the trailing edge of the aerofoil are planned to enhance the image processing and for a better understanding of the atomization at high speed flows.

We gratefully acknowledge the support by the Grant Agency of the Czech Technical University in Prague, Grant No. SGS 13/18 and Grant No. 16-20123S of the Grant Agency of the Czech Republic.

\section{References}

1. O. Bartoš, X. Cai, M. Kolovratník, EPJ Web of Conferences. 2014, vol.. 67, ISSN 2100-014X

2. M. Kolovratník, O. Bartoš, EPJ Web of Conferences, 92 (2015) 02035 ISSN 2100-014X.

3. M.J. Moore, C.H. Sieverding, Two - Phase Steam Flow in Turbines and Separators, McGraw-Hill, 1976.

4. J. Valha, Proudění mokré páry a její erozivní účinky V průtočné části parní turbíny. SVUSS, Sborník referátu, 1978.

5. H. Lange, Dynamic surface tension of detergent solutions. Jou. of Colloid Science, 1965. 